\title{
Exploring the explosion mechanism of core-collapse supernovae in three dimensions
}

\author{
Tobias Melson ${ }^{1}$, Hans-Thomas Janka ${ }^{1}$, Alexander Summa ${ }^{1}$, \\ Robert Bollig $^{1}$, Andreas Marek ${ }^{2}$ and Bernhard Müller ${ }^{3,4}$ \\ ${ }^{1}$ Max-Planck-Institut für Astrophysik, \\ Karl-Schwarzschild-Str. 1, 85748 Garching, Germany \\ ${ }^{2}$ Max Planck Computing and Data Facility (MPCDF), \\ Gießenbachstr. 2, 85748 Garching, Germany \\ ${ }^{3}$ Astrophysics Research Center, School of Mathematics and Physics, Queen's University, \\ Belfast BT7 1NN, United Kingdom \\ ${ }^{4}$ Monash Center for Astrophysics, School of Physics and Astronomy, \\ Building 79P, Monash University, Victoria 3800, Australia
}

\begin{abstract}
We present the first successful simulations of neutrino-driven supernova explosions in three dimensions (3D) using the Vertex-Prometheus code including sophisticated energydependent neutrino transport. The simulated models of 9.6 and 20 solar-mass iron-core stars demonstrate that successful explosions can be obtained in self-consistent 3D simulations, where previous models have failed. New insights into the supernova mechanism can be gained from these explosions. The first 3D model (Melson et al. 2015a) explodes at the same time but more energetically than its axially symmetric (2D) counterpart. Turbulent energy cascading reduces the kinetic energy dissipation in the cooling layer and therefore suppresses neutrino cooling. The consequent inward shift of the gain radius increases the gain layer mass, whose recombination energy provides the surplus for the explosion energy.

The second explosion (Melson et al. 2015b) is obtained through a moderate reduction of the neutral-current neutrino opacity motivated by strange-quark contributions to the nucleon spin. A corresponding reference model without these corrections failed, which demonstrates how close current 3D models are to explosion. The strangeness adjustment is meant as a prototype for remaining neutrino opacity uncertainties.
\end{abstract}

Keywords. supernovae: general, neutrinos, hydrodynamics, turbulence

\section{References}

Melson, T., Janka, H.-T., \& Marek, A. 1995, ApJ (Letters), 801, L24

Melson, T., Janka, H.-T., Bollig, R., Hanke, F., Marek, A., \& Müller, B. 2015, ApJ (Letters), $808, \mathrm{~L} 42$ 